\title{
Parthenogenesis in Human Oocytes that Were Collected From Resected Ovarian Tissue and Matured In Vitro
}

\author{
Ho-Joon Lee and Jose Teixeira
}

Currently, a major hurdle in the progress of human embryonic stem (hES) cell research is the lack of human oocytes with which to perform experiments. The collection process is a logistical and ethical challenge and usually involves the use of excess oocytes donated after assisted reproduction procedures. We collected resected human ovarian tissue after routine surgical procedures. Oocytes were isolated from the tissue and matured in vitro to the meiosis II (MII) stage, when the first polar body is extruded. With the large antral and smaller preantral follicles, the efficiencies of the maturation were nearly $50 \%$ and $25 \%$, respectively. The quality of the matured oocytes was assessed by inducing parthenogenesis and $>50 \%$ of the in vitro matured oocytes were competent enough to develop pronuclei and 33\% developed at least to the two-cell stage $48 \mathrm{~h}$ after activation. Parthenotes continued to develop by $72 \mathrm{~h}$ but with significant blastomere fragmentation. These results provide evidence that resected ovarian tissue, which is normally discarded, may be a suitable alternative source for oocytes in hES cell research.

\section{Introduction}

$\mathrm{T}$ HE POSSIBILITY THAT EMBRYONIC stem cells could be used to replace diseased, damaged, or lost cells promises to revolutionize how medicine is practised in the future. Human embryonic stem (hES) cells are largely derived from surplus blastocysts but in order to realize the full potential of hES therapy, the cells must be recognized as self by the transplant recipient [1]. Expression of key master transcriptional regulators can induce pluripotency in a patient's own formerly differentiated cells and may be a mechanism to create histocompatible hES cells [2-4]. However, the viral transduction delivery system currently used to express those genes renders the resulting induced pluripotent stem (iPS) cells unsuitable for therapeutic use until safer delivery methods are developed [5]. Somatic cell nuclear transfer (SCNT) is another mechanism that has been proposed to make patientspecific embryonic stem cells. Using this method, the endogenous nuclear material from donor human oocytes would be replaced with that of one of the patient's cells and then the renucleated oocyte would be stimulated to divide and develop for the isolation of the self-hES cells [6-8].

In order for progress to be made in SCNT research, a steady supply of human oocytes is needed. However, acquiring sufficient oocytes from donors undergoing requisite fertility treatments with no reproductive or medical benefit to themselves is problematic for both ethical and logistical reasons. Oocyte donation is a grueling process involving daily injections over 7-10-day period followed by ultrasound-guided, transvaginal follicle aspiration under anesthesia. There is a significant risk of severe ovarian hyperstimulation syndrome to the donor with some reports indicating that up to $10 \%$ of oocyte donors experience symptoms, including pain sufficient to cause hospitalization, renal failure, potential future infertility, and death [9].

Oocytes mature in vivo during a poorly understood process called folliculogenesis. At puberty the ovary contains $\sim 150,000$ oocytes; most will undergo atresia and a few hundred will ovulate throughout the reproductive life span of an individual [10]. Primordial and primary oocytes persist in meiotic prophase and reside in the cortical region of the ovary. Once selected during folliculogenesis, these primary oocytes proceed through several stages of maturation in follicles, generally defined as preantral, early antral, antral, or Graffian follicles. In vivo, it takes many months for a recruited primordial follicle to develop into a preovulatory follicle. There are a number of reports describing the in vivo and in vitro maturation (IVM) of oocyte from human ovarian tissue [11-15].

Vincent Center for Reproductive Biology, Department of Obstetrics, Gynecology, and Reproduction, Vincent Memorial Hospital, Massachusetts General Hospital and Harvard Medical School, Boston, Massachusetts. 
A recent report showed that activin, a member of the transforming growth factor- $\beta$ (TGF $\beta$ ) family that promotes follicle-stimulating hormone $(\mathrm{FSH})$ release from the pituitary, is also produced by granulosa cell (GC) and can induce follicle growth on its own in vitro [16]. FSH induces follicular maturation and is widely used in fertility centers to increase available oocytes for in vitro fertilization. Müllerian inhibiting substance (MIS, also known as anti-Müllerian hormone or $\mathrm{AMH}[17,18])$ is another member of the TGF $\beta$ family of growth and differentiation factors. Its main function is to induce regression of the Müllerian duct in male fetuses but it is also produced by the endogenous GCs of developing follicles in the ovary and appears to inhibit early follicular development both in vivo [19] and meiosis II (MII) in vitro [20]. The utility of manipulating MIS activity in IVM has not been described.

Together with the recent progress reported with IVM of human oocytes and subsequent successful fertilization and pregnancy in IVF protocols [21-23], we hypothesized that oocytes from discarded ovarian tissue of patients undergoing routine oophorectomy may be a feasible alternative to oocyte donation. In this report, we show that preantral and antral follicles collected from resected human ovarian tissue can be matured in vitro and stimulated to undergo parthenogenesis.

\section{Materials and Methods}

\section{Ovarian tissues}

Ovarian tissue was collected for these studies from consented patients undergoing routine oophorectomy for benign disease or endometrial cancer using MGH IRBapproved protocols. The patients were premenopausal, with ages ranging from 26 to 53, with a mean of 42.3, a median of 43 , and a standard deviation of 6.1. Excess ovarian tissue was transported from pathology to the laboratory immediately. The medullar part was removed from the biopsies using microsurgical scissors. Antral follicles in the cortex of ovarian tissues were punctured with a $27 \mathrm{G}$ needle to collect oocytes and the remaining cortical strips were cut into approximately $2-3 \mathrm{~mm}^{3}$ for subsequent experiments.

\section{Ovarian tissue culture and follicle counting}

Ten biopsy fragments from each patient ( $n=5$ patients, total 50 fragments) were placed in a six-well plate using Transwell culture dishes (Corning, Corning, NY) containing $3 \mathrm{~mL}$ of culture media of Dulbecco's modified eagle's medium (DMEM)/F12 and 10\% fetal bovine serum (FBS) (Invitrogen, Carlsbad CA) containing five different groups: (1) medium with unsexed FBS, (2) medium with female FBS (Aires Scientific/Biologos, Richardson, TX), (3) medium with MIS (5 $\mu \mathrm{g} / \mathrm{mL})$, (4) medium with an anti-MIS antibody ( $\alpha$-MIS, 1:200) (both MIS and the MIS antibody were kind gifts from Dr. David T. MacLaughlin, MGH, Boston, MA), and (5) medium with FSH/LH 1 IU/mL (Ferring Pharmaceuticals, Parsippany, NJ), each with supplemented 1\% (vol/vol) Insulin, Transferrin, Selenium (Invitrogen), 1\% (vol/vol) penicillin G, streptomycin, glutamine, (Invitrogen) for 7 and 14 days culture.

\section{Histological evaluation}

Five random pieces of biopsies in each group were taken and fixed overnight in Bouin's solution for histological evaluation at 7 and 14 days. Fixed tissues were dehydrated in a graded ethanol process using a tissue processor (Leica Microsystems, Bannockburn, IL) and individual pieces were embedded in paraffin wax. All samples were completely sectioned at a thickness of $8 \mu \mathrm{m}$, mounted on slides, dried overnight at room temperature, and stained with hematoxylin and eosin. Each section was examined by light microscopy and follicles containing an oocyte nucleolus were counted and classified. Follicles were classified from a minimum of three patients by morphology based on oocyte surrounded GC as follows: (1) primordial follicle: oocyte surrounded by one flattened GC layer; (2) first primary follicle: oocyte surrounded by two flattened and cuboidal layers; (3) second primary follicle: oocyte surrounded by two cuboidal GC layers; (4) preantral follicle: oocytes surrounded by multiple GC layers; and (5) antral follicle: presence of antral cavity within follicle.

\section{Follicle isolation}

About 20 biopsy fragments from any given patient were placed in center-well organ culture dish (Falcon, Franklin Lakes, NJ) containing $1 \mathrm{~mL}$ of DMEM/F12 medium supplemented with $1 \mathrm{mg} / \mathrm{mL}$ collagenase type IA (Invitrogen) and incubated for $2 \mathrm{~h}$ in $5 \% \mathrm{CO}_{2}$ in a humidified atmosphere. After enzymatic digestion, enzymatic solution was mixed and washed by an equal volume of culture media. The suspension was centrifuged twice at $100 \mathrm{~g}$ for $5 \mathrm{~min}$ at $4^{\circ} \mathrm{C}$. The supernatant was then discarded and the pellet was transferred to Petri dishes and investigated for follicles under a stereomicroscope (TE2000S; Nikon Instruments, Japan). The follicles were collected, washed with PBS medium supplemented with $10 \%$ FBS, and placed in culture media.

\section{Collection of mature oocytes and parthenogenetic activation}

Isolated follicles were cultured in a six-well plate using Transwell culture dishes containing $3 \mathrm{~mL}$ culture media with $1 \mathrm{IU} / \mathrm{mL}$ FSH/LH for 7-14 days. Mature oocytes were placed in HTF medium with 10\% serum substitute supplement (SSS) (Irvine Scientific, Santa Ana, CA). For activation, mature oocytes were cultured for $6 \mathrm{~h}$ in $\mathrm{Ca}^{2+}$-free M16 (Invitrogen) supplemented with $10 \mathrm{mM} \mathrm{SrCl}_{2}$ (Sigma, St. Louis, MO) and $5 \mu \mathrm{g} / \mathrm{mL}$ cytochalasin B (Sigma). After activation, oocytes were further cultured in $50 \mu \mathrm{L}$ droplets of HTF with $10 \%$ SSS covered mineral oil (Irvine) in $5 \% \mathrm{CO}_{2}$ at $37^{\circ} \mathrm{C}$ in a humidified atmosphere. Oocytes were evaluated every $24 \mathrm{~h}$ after activation.

\section{Statistical methods}

Data were analyzed by ANOVA with Tukey's Multiple Comparison Test using Prism software (GraphPad Software, LaJolla, CA).

\section{Results}

The first set of human ovarian tissue specimens we collected were used to assess the feasibility of using resected ovarian tissue for oocyte collection and maturation. The ovarian cortical tissue from five patients was collected and cut into 2-3 $\mathrm{mm}^{3}$ pieces and the number and quality of the oocytes 
found was determined by histological analyses. Figure $1 \mathrm{~A}-\mathrm{D}$ shows representative photomicrographs of primordial, primary, preantral, and antral follicles. The scarcity of follicles compared to that of a younger ovary was the most striking feature we observed. In general, the oocytes looked normal and we observed very few atretic follicles (data not shown). The results of counting follicles in the ovarian are shown in Table 1 . There was great variability in the total number of oocytes from patient to patient and between ovarian pieces from the same patient. There were very few preantral and antral follicles, which were fairly consistent between patients.

Since the number of the larger mature follicles per 2-3 $\mathrm{mm}^{3}$ section of ovary was only 1 or 2 , we next attempted to increase their numbers by maturation of primary and preantral follicles in media with hormonal conditions known to affect folliculogenesis (Fig. 2). In a series of experiments, we compared folliculogenesis in organ culture of ovarian tissue fragments $(n=5)$ to determine whether MIS, like activin, could promote follicular development with media that either contained female fetal calf serum to remove any possible inhibitory effects of MIS or normal mixed male and female fetal calf serum with the addition of either FSH to stimulate folliculogenesis, or an antibody to MIS to inhibit the binding of both the endogenously produced MIS and the bovine serum MIS, or the MIS ligand itself as a control (Fig. 2). Preantral follicles were not observed in the ovarian organ cultures incubated with MIS. Ovarian tissue fragments incubated with MIS antibodies yielded more preantral follicles after 7 days in culture. Incubation with anti-MIS for 2 weeks reversed that effect. The greatest increase in the number of preantral follicles was observed with fragments incubated with FSH after 14 days in culture. These results suggested that organ culture of ovarian tissue fragments with MIS antibodies would be a feasible mechanism for increasing the number of mature antral follicles but that FSH alone was best.

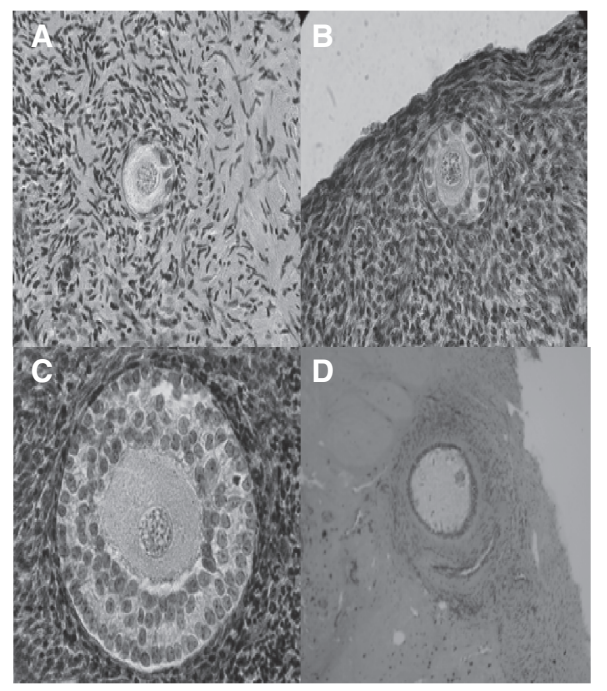

FIG. 1. Histology of follicles collected from discarded human ovary. (A) Primordial follicle surrounded by one layer of granulosa cells. (B) Primary follicle with two layers of granulosa cells. (C) Preantral follicle with multiple layers of granulosa cells. (D) Small antral follicle with a developing antrum and several layers of granulosa cells.
In the next set of experiments, follicles were released from the ovarian stroma in the tissue fragments by enzymatic digestion of the smaller follicles (Fig. $3 \mathrm{~A}$ and $\mathrm{B}$ ) and by mechanical puncture of the larger follicles (Fig. 3C and D). The follicle recovery rates from the ovarian tissue fragments are shown in Table 2. Whereas the number of primordial follicles recovered by this method was significantly less ( 3 vs. $20, P<0.001$ ) from that which is normally found in the ovarian fragments, the number of larger follicles isolated was not significantly different from the number found in the untreated normal ovarian fragments shown in Table 1, indicating that isolation of follicles by mechanical puncture and enzymatic digestion would be an efficient method to isolate primary through antral follicles as cumulous oocyte complexes (COCs; Fig. 3E and F).

We next incubated the isolated follicles with FSH until the first polar body was extruded by the oocyte, which usually required incubation between 7 and 14 days. The results are shown in Table 3 . We were able to successfully mature the larger preantral and antral follicles, $26 \%$ and $46 \%$, respectively, which were usually isolated as COCs, to the MII stage (Fig. 4A). However, none of the smaller primordial or primary follicles were matured in vitro using these methods. In order to assess the quality of the in vitro matured MII oocytes, we induced the oocytes to undergo parthenogenesis with strontium chloride and cytocholasin B. From the 12 MII oocytes that were matured in vitro, a total of seven parthenotes were observed with pronuclei $(\mathrm{PN})$ within 48 $\mathrm{h}$ after stimulation. The remaining oocytes either did not develop pronuclei or were degenerating. Parthenotes may also develop with one or multiple pronuclei, but those with two pronuclei have been reported to have the best outcome in blastocyst development [24]. A typical parthenote with two pronuclei that developed during this study is shown in Figure 4B. Three of the parthenotes developed to the twocell stage (Fig. 4C). After $72 \mathrm{~h}$, the parthenotes began to show significant levels of blastomere fragmentation. Figure 4D shows one of the degenerating parthenotes with three otherwise intact blastomeres.

\section{Discussion}

In a study using surplus IVF oocytes, the efficiency of parthenogenesis by activation of normal, human MII oocytes has been reported to be as high as $67 \%$ [25]. We show that the efficiency of activation of in vitro matured MII oocytes is compromised compared to that of IVF oocytes, but still

Table 1. The Number of Follicles in Discarded Ovary Fragments by Histology

\begin{tabular}{lccccc}
\hline & \multicolumn{5}{c}{ Follicle } \\
\cline { 2 - 6 } Patient & Primordial & Primary & Preantral & Antral & Total \\
\hline CP1 & 9 & 17 & 0 & 2 & 28 \\
CP2 & 20 & 21 & 1 & 1 & 43 \\
CP3 & 43 & 29 & 1 & 2 & 75 \\
CP5 & 11 & 12 & 1 & 1 & 25 \\
CP7 & 16 & 8 & 0 & 0 & 23 \\
Average & 20 & 17 & 0.6 & 1.2 & 39 \\
\hline
\end{tabular}



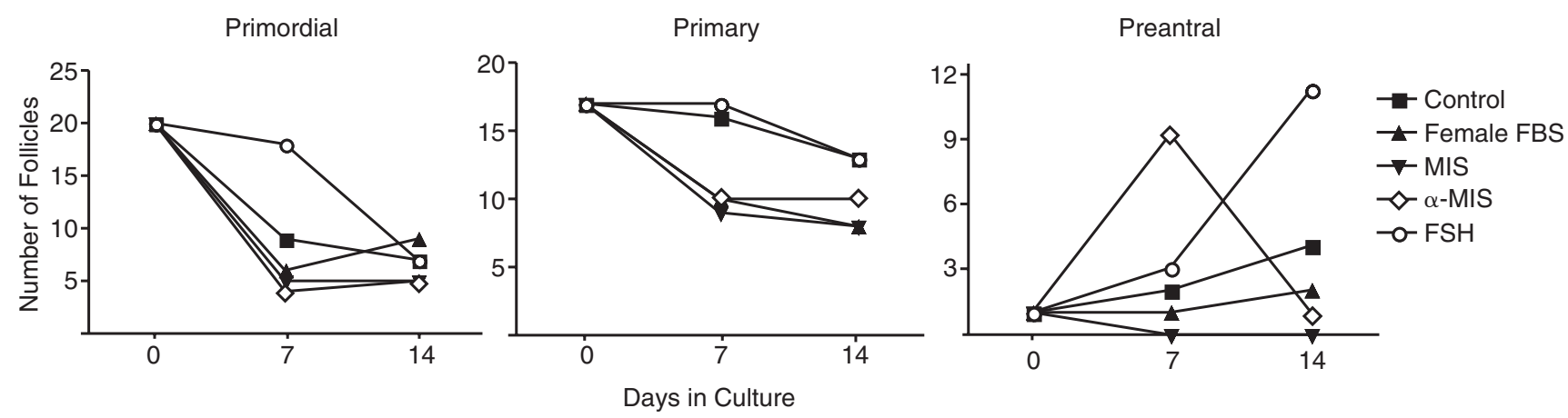

FIG. 2. In vitro maturation of follicles. Human ovarian fragments ( $n=5$ in each group) were incubated in control media with normal FBS, medium with female FBS that lacks MIS, medium with normal FBS and added recombinant human MIS, medium with normal FBS and an antibody to MIS, and medium with normal FBS and added FSH. The tissue fragments were allowed to incubate for 7 or 14 days before counting and grading the number of follicles in each fragment. The total number of each follicle in each group is shown plotted.

compares favorably, at $33 \%$. However, while the rate efficiency of blastocyst formation in that study was 13\% [25], we did not observe blastocyst formation from any of the activated oocytes that were matured in vitro. These results suggest that in vitro matured human oocytes may not be competent to develop past early cleavage embryos after parthenogenic activation. Or else activation of the oocyte by sperm introduces factors that are needed for in vitro matured oocytes to develop into blastocysts that are not needed for in vivo matured oocytes.

This latter hypothesis is further supported by recent results with adult murine oocytes in which there were no blastocysts observed after in vitro maturation and parthenogenic activation [26]. In contrast, $40 \%$ of similarly matured mouse oocytes fertilized in vitro developed into blastocysts. Another study by the same group showed that $20 \%$ of oocytes collected and matured in vitro from juvenile mice and

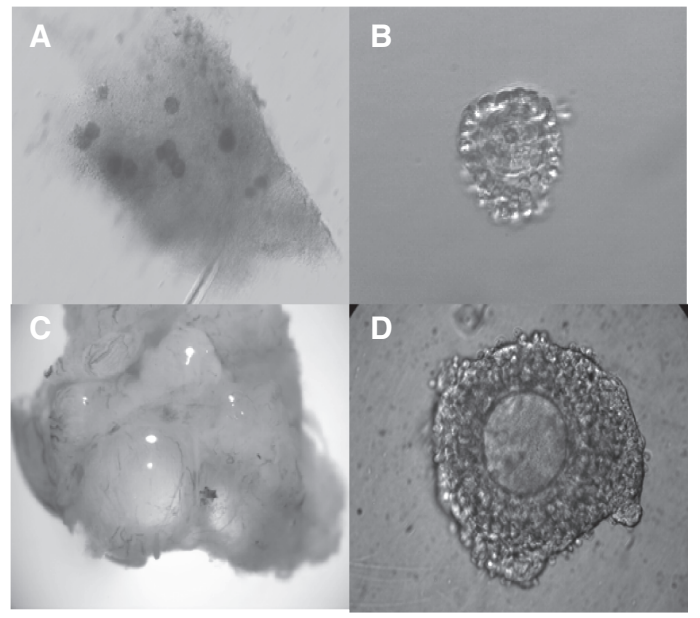

FIG. 3. Representative isolated follicles from discarded ovarian tissue are shown. Enzymatic digestion results in the release of many primordial and primary follicles (A and B), whereas mechanical puncture results in the release of predominantly larger follicles (C and D). activated by parthenogenesis could develop into blastocysts [27], suggesting that oocytes from immature ovaries could overcome the lack of a sperm-specific requirement of oocytes from mature ovaries. Neither the identity of the sperm-specific protein or factor required for overcoming the arrest in blastocyst development from parthenogenetically activated adult oocytes nor the post two-cell arrest mechanism itself is known. The hormonal control of oocyte maturation does not seem to significantly contribute to their competence after parthenogenetic activation since juvenile in vitro matured murine oocytes can develop into blastocysts after activation [27] suggesting that the culture conditions may not be the impediment. Therefore, one could speculate that the hypothetical blastocyst arrest factor must be intrinsic to in vitro matured oocytes. Alternatively, the loss of developmental competence by IVM oocytes could be a trivial decoupling of normally coordinated nuclear and cytoplasmic development

Table 2. The Number of Follicles Isolated From Discarded Ovary Fragments

\begin{tabular}{lccccc}
\hline & \multicolumn{5}{c}{ Follicle } \\
\cline { 2 - 6 } Patient & Primordial & Primary & Preantral & Antral & Total \\
\hline CP11 & 0 & 5 & 0 & 1 & 6 \\
CP12 & 0 & 10 & 2 & 1 & 13 \\
CP21 & 2 & 14 & 2 & 1 & 19 \\
CP22 & 3 & 12 & 2 & 0 & 17 \\
CP23 & 3 & 12 & 0 & 0 & 15 \\
CP28 & 0 & 5 & 2 & 0 & 7 \\
CP35 & 3 & 3 & 2 & 3 & 11 \\
CP38 & 1 & 2 & 1 & 1 & 5 \\
CP39 & 3 & 3 & 1 & 0 & 7 \\
CP41 & 1 & 3 & 1 & 0 & 5 \\
CP42 & 4 & 5 & 3 & 0 & 12 \\
CP43 & 10 & 10 & 2 & 0 & 22 \\
CP47 & 8 & 12 & 2 & 2 & 24 \\
CP49 & 5 & 3 & 2 & 0 & 10 \\
CP50 & 3 & 7 & 5 & 2 & 17 \\
Average & 3 & 7 & 1.8 & 0.7 & 12 \\
\hline
\end{tabular}


Table 3. Follicle and Oocyte Maturation by in vitro Culture Followed by PARTHENOGENESIS

\begin{tabular}{|c|c|c|c|c|c|c|c|}
\hline \multirow[b]{2}{*}{ Follicle } & \multicolumn{4}{|c|}{ In vitro maturation } & \multicolumn{3}{|c|}{ Parthenogenesis } \\
\hline & Total & Atretic & Immature & $\begin{array}{l}\text { Mature } \\
(\% \text { total })\end{array}$ & $\begin{array}{c}P N \\
(\% \text { total })\end{array}$ & $\begin{array}{l}>2 \text { Cell } \\
(\% \text { total })\end{array}$ & Degeneration \\
\hline Primordial & 46 & 46 & 0 & 0 & 0 & 0 & 0 \\
\hline Primary & 106 & 86 & 20 & 0 & 0 & 0 & 0 \\
\hline Preantral & 27 & 12 & 8 & $7(26)$ & 3 & 1 & 3 \\
\hline Antral & 11 & 3 & 3 & $5(46)$ & 1 & 2 & 2 \\
\hline Total & 190 & 147 & 31 & $12(6)$ & $4(33)$ & $3(25)$ & 5 \\
\hline
\end{tabular}

in vivo [15] with observable nuclear development indicated by polar body extrusion without the requisite corresponding cytoplasmic development.

Clinically, the utility of IVM for patients with polycystic ovarian disease and at risk for hyperstimulation has been described (reviewed in [28]). IVM from retrieved GV oocytes has been used to improve the reproductive outcome of these patients indicating that IVM from late meiosis I may not be compromised to the same degree as with IVM of earlier stage oocytes. However, blastocyst development from fertilized IVM oocytes is not as efficient as in vivo matured oocytes suggesting that in addition to a developmental factor, IVM culture conditions may not yet be optimal.

We have shown that human oocytes collected from resected ovarian tissue can be matured in vitro and can be activated to develop as parthenotes at least to the two-cell stage. Our attempts to improve the number of later stage

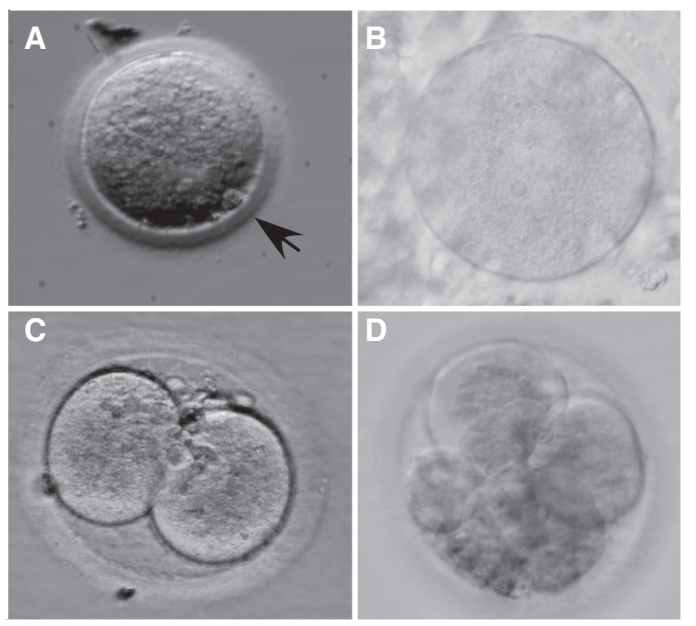

FIG.4. In vitro maturation and parthenogenesis of oocytes collected from resected ovarian tissue. A typical meiosis II (MII) oocyte matured in vitro with a prominent polar body (arrow) is shown in A. After activation of an MII oocyte by strontium chloride and cytocholasin B, parthenogenesis begins with development of two pronuclei (B). The following day parthenogenesis proceeds with the development of two blastomeres (C). After the two-cell stage, some of the blastomeres in all the parthenotes showed signs of degeneration $(\mathbf{D})$. oocytes by incubating ovarian tissue with antibodies to MIS, an in vivo inhibitor of follicle recruitment [29], met with limited success when compared to FSH alone. However, our data suggests that the induction of oocyte development in the first week of culture by inhibiting MIS signaling followed by a second week of incubation with FSH and mechanical disruption might prove beneficial. Studies are underway to develop this hypothesis further so that the maximum number of more mature oocytes can be culled from any given ovarian tissue fragment.

Our results present two opportunities for using IVM oocytes in hES cell research. The parthenotes we derived from IVM oocytes showed significant blastomere fragmentation after the two-cell stage, which is known to render the embryos less likely to develop to the blastocyst stage. Defragmentation of mouse embryos with significant blastomere fragmentation has been used to rescue the embryos, allowing them to develop to the blastocyst stage [30]. Similar defragmentation of the parthenotes might also render them amenable to further development and hES cell derivation. Alternatively, a number of groups have reported derivation of ES cells from a single mouse or human blastomere [31-34]. We have shown that $25 \%$ of the parthenotes developing from in vitro matured oocytes have at least two blastomeres, which could also be used in single cell-mediated derivation protocols. However, identification of the molecular mechanisms blocking blastocyst development after two-cell stage will be critical for overcoming this hurdle and the use of in vitro matured oocytes in hES research using standard techniques of derivation from the inner cell mass.

\section{Acknowledgments}

These studies were partially supported by the Harvard Stem Cell Institute and the Stem Cell Research Foundation grants to JT. We would like to thank Audrey Etlinger for consenting patients and collecting ovarian tissue for this study. We would also like to acknowledge the generous support of Vincent Memorial Research Funds.

\section{Author Contributions}

Ho-Joon Lee: Conception and design, collection and/or assembly of data, data analysis and interpretation, manuscript writing, and final approval.

Jose Teixeira: Conception and design, data analysis and interpretation, manuscript writing, and final approval. 


\section{References}

1. Wobus AM and KR Boheler. (2005). Embryonic stem cells: prospects for developmental biology and cell therapy. Physiol Rev 85:635-678.

2. Takahashi K and S Yamanaka. (2006). Induction of pluripotent stem cells from mouse embryonic and adult fibroblast cultures by defined factors. Cell 126:663-676.

3. Wernig $\mathrm{M}, \mathrm{A}$ Meissner, $\mathrm{R}$ Foreman, $\mathrm{T}$ Brambrink, $\mathrm{M} \mathrm{Ku}, \mathrm{K}$ Hochedlinger, BE Bernstein and R Jaenisch. (2007). In vitro reprogramming of fibroblasts into a pluripotent ES-cell-like state. Nature 448:318-324.

4. Yu J, MA Vodyanik, K Smuga-Otto, J Antosiewicz-Bourget, JL Frane, S Tian, J Nie, GA Jonsdottir, V Ruotti, R Stewart, Slukvin II, and JA Thomson. (2007). Induced pluripotent stem cell lines derived from human somatic cells. Science 318:1917-1920.

5. Yu J and JA Thomson. (2008). Pluripotent stem cell lines. Genes Dev 22:1987-1997.

6. Mai Q, Y Yu, T Li, L Wang, MJ Chen, SZ Huang, C Zhou and Q Zhou. (2007). Derivation of human embryonic stem cell lines from parthenogenetic blastocysts. Cell Res 17:1008-1019.

7. Revazova ES, NA Turovets, OD Kochetkova, LS Agapova, JL Sebastian, MV Pryzhkova, VI Smolnikova, LN Kuzmichev and JD Janus. (2008). HLA homozygous stem cell lines derived from human parthenogenetic blastocysts. Cloning Stem Cells 10:11-24.

8. Revazova ES, NA Turovets, OD Kochetkova, LB Kindarova, LN Kuzmichev, JD Janus and MV Pryzhkova. (2007). Patientspecific stem cell lines derived from human parthenogenetic blastocysts. Cloning Stem Cells 9(3):432-439.

9. Magnus D and MK Cho. (2005). Ethics. Issues in oocyte donation for stem cell research. Science 308:1747-1748.

10. Gougeon A. (1986). Dynamics of follicular growth in the human: a model from preliminary results. Hum Reprod 1:81-87.

11. Abir R, S Nitke, A Ben-Haroush and B Fisch. (2006). In vitro maturation of human primordial ovarian follicles: clinical significance, progress in mammals, and methods for growth evaluation. Histol Histopathol 21:887-898.

12. Gook DA, DH Edgar, J Borg, J Archer, PJ Lutjen and JC McBain. (2003). Oocyte maturation, follicle rupture and luteinization in human cryopreserved ovarian tissue following xenografting. Hum Reprod 18:1772-1781.

13. Gosden RG, J Mullan, HM Picton, H Yin and SL Tan. (2002). Current perspective on primordial follicle cryopreservation and culture for reproductive medicine. Hum Reprod Update 8:105-110.

14. Oktay K and M Sonmezer. (2007). Fertility preservation in gynecologic cancers. Curr Opin Oncol 19:506-511.

15. Trounson A, C Anderiesz and G Jones. (2001). Maturation of human oocytes in vitro and their developmental competence. Reproduction 121:51-75.

16. Telfer EE, M McLaughlin, C Ding and KJ Thong. (2008). A two-step serum-free culture system supports development of human oocytes from primordial follicles in the presence of activin. Hum Reprod 23:1151-1158.

17. Josso N, JY Picard, R Rey and N di Clemente. (2006). Testicular anti-Mullerian hormone: history, genetics, regulation and clinical applications. Pediatr Endocrinol Rev 3:347-358.

18. Teixeira J, S Maheswaran and PK Donahoe. (2001). Müllerianinhibiting substance: an instructive developmental hormone with diagnostic and possible therapeutic applications. Endocr Rev 22:657-674.

19. Durlinger AL, P Kramer, B Karels, FH de Jong, JT Uilenbroek, JA Grootegoed and AP Themmen. (1999). Control of primordial follicle recruitment by anti-Müllerian hormone in the mouse ovary. Endocrinology 140:5789-5796.

20. Takahashi M, SS Koide and PK Donahoe. (1986). Müllerian inhibiting substance as oocyte meiosis inhibitor. Mol Cell Endocrinol 47:225-234.
21. Chian RC. (2004). In-vitro maturation of immature oocytes for infertile women with PCOS. Reprod Biomed Online 8:547-552.

22. Kanaya H, Y Murata, H Oku, S Hashimoto, $\mathrm{Y}$ Morimoto, $\mathrm{T}$ Murata, K Sugihara, F Nagata, Y Nakaoka and A Fukuda. (2006). Successful monozygotic twin delivery following in vitro maturation of oocytes retrieved from a woman with polycystic ovary syndrome: case report. Hum Reprod 21:1777-1780.

23. Kyono K, N Fukunaga, K Haigo, S Chiba, C Ohira, Y Nakajo and Y Araki. (2002). Successful delivery following cryopreservation of zygotes produced by in vitro matured oocytes retrieved from a woman with polycystic ovarian syndrome-like disease: a case report. J Assist Reprod Genet 19:390-393.

24. Lee JW, XC Tian and X Yang. (2004). Optimization of parthenogenetic activation protocol in porcine. Mol Reprod Dev 68:51-57.

25. Paffoni A, TA Brevini, E Somigliana, L Restelli, F Gandolfi and G Ragni. (2007). In vitro development of human oocytes after parthenogenetic activation or intracytoplasmic sperm injection. Fertil Steril 87:77-82.

26. Kim IW, SP Gong, CR Yoo, JH Choi, DY Kim and JM Lim. (2008). Derivation of developmentally competent oocytes by the culture of preantral follicles retrieved from adult ovaries: maturation, blastocyst formation, and embryonic stem cell transformation. Fertil Steril. [Epub ahead of print]

27. Lee ST, MH Choi, EJ Lee, SP Gong, M Jang, SH Park, H Jee, DY Kim, JY Han and JM Lim. (2008). Establishment of autologous embryonic stem cells derived from preantral follicle culture and oocyte parthenogenesis. Fertil Steril 90:1910-1920.

28. Reinblatt SL and W Buckett. (2008). In vitro maturation for patients with polycystic ovary syndrome. Semin Reprod Med 26:121-126.

29. Durlinger AL, MJ Gruijters, P Kramer, B Karels, TR Kumar, MM Matzuk, UM Rose, FH de Jong, JT Uilenbroek, JA Grootegoed and AP Themmen. (2001). Anti-Mullerian hormone attenuates the effects of FSH on follicle development in the mouse ovary. Endocrinology 142:4891-4899.

30. Alikani M, F Olivennes and J Cohen. (1993). Microsurgical correction of partially degenerate mouse embryos promotes hatching and restores their viability. Hum Reprod 8:1723-1728.

31. Chung Y, I Klimanskaya, S Becker, T Li, M Maserati, SJ Lu, T Zdravkovic, D Ilic, O Genbacev, S Fisher, A Krtolica and R Lanza. (2008). Human embryonic stem cell lines generated without embryo destruction. Cell Stem Cell 2:113-117.

32. Feki A, A Bosman, JB Dubuisson, O Irion, S Dahoun, MF Pelte, O Hovatta and ME Jaconi. (2008). Derivation of the first Swiss human embryonic stem cell line from a single blastomere of an arrested four-cell stage embryo. Swiss Med Wkly 138:540-550.

33. Klimanskaya I, Y Chung, S Becker, SJ Lu and R Lanza. (2007). Derivation of human embryonic stem cells from single blastomeres. Nat Protoc 2:1963-1972.

34. Lorthongpanich C, SH Yang, K Piotrowska-Nitsche, R Parnpai and AW Chan. (2008). Development of single mouse blastomeres into blastocysts, outgrowths and the establishment of embryonic stem cells. Reproduction 135:805-813.

Address correspondence to: Dr. Jose Teixeira Vincent Center for Reproductive Biology/Thier 913 Massachusetts General Hospital and Harvard Medical School 55 Fruit Street Boston, MA 02114

E-mail: teixeira@helix.mgh.harvard.edu

Received for publication January 5, 2009 Accepted after revision February 27, 2009 Prepublished on Liebert Instant Online February 27, 2009 